\title{
Integral membrane protein expression of human CD25 on the cell surface of HEK293 cell line: the available cellular model of $C D 25$ positive to facilitate in vitro developing assays
}

https://doi.org/10.1515/bmc-2019-0018

received April 29, 2019; accepted July 4, 2019.

\begin{abstract}
Typically, CD25 is expressed on the cellular surface of regulatory $\mathrm{T}$ (Treg) cells. These cells are significant in regulating the self-tolerance and also preventing the immune system from attacking a person's own tissues and cells. They promote the cancer progression by playing an important role in evading the immune system. Thus, the experimental procedures was aimed to clone and express human CD25 in HEK293 cell line, as the available cellular model, for the purpose of developing assays to facilitate and enhance the studies on an available CD25 positive cell. The secondary RNA structure of CD25 was evaluated by in silico analysis. Then, cDNA of human CD25 were synthesized from isolated total mRNA of cultured and stimulated PBMCs from blood donors. After cloning the cDNA of CD25 into a pcDNA3.1(+) plasmid, using the effective transfection of the recombinant pcDNA3.1(+) in HEK293, qRT-PCR and flow cytometry methods were used to quantitatively evaluate CD25 transcripts and protein level. There was a
\end{abstract}

\footnotetext{
*Corresponding authors: Zohreh Hojati, Division of Genetics, Department of Biology, Faculty of Sciences, University of Isfahan, Postal Code: 81746-73441, Isfahan, Iran, E-mail: z.hojati@sci.ui.ac.ir; Mazdak Ganjalikhani-Hakemi, Department of Immunology, Faculty of Medicine, Isfahan University of Medical Sciences, Postal Code: 81746-73461, Isfahan, Iran. Acquired Immunodeficiency Research Center, Isfahan University of Medical Sciences, Isfahan, Iran, E-mail: mghakemi@med.mui.ac.ir

Moein Dehbashi, Division of Genetics, Department of Biology, Faculty of Sciences, University of Isfahan, Postal Code: 81746-73441, Isfahan, Iran

Majid Motovali-bashi, Division of Genetics, Department of Biology, Faculty of Sciences, University of Isfahan, Postal Code: 81746-73441 Isfahan, Iran

Akihiro Shimosaka, Institute of Hematology, Peking Union Medical College, Beijing, China
}

4.8 fold increase in transcripts and a 76.2\% increase in protein levels of CD25 when comparing the transfected and control cell lines. The genetically engineered HEK293 cell line expressing Treg cell surface marker of CD25 was introduced in this study for the first time. This cell line can be used to overcome the problematic issues for studying Treg cells including low population of Tregs in peripheral blood, low recovery methods for Treg isolation, timeconsuming and non-cost benefit methods in the conditions of in vitro cell culture experiments for the studies focused on the binding of IL-2 to CD25.

Keywords: CD25; HEK293; Transfection; qRT-PCR; Flow cytometry

\section{Introduction}

Nowadays, cancers pose the major challenges for human health. During carcinogenesis, elimination of the tumor through the activity of the immune system seldom arises, despite an expansion of the immune response. The use of immunotherapy to power the common tumor particular immune response has restricted clinical usage, since cancerous cells possess a diversity of tactics to evade immune surveillance ${ }^{1}$. Evading the identification and regulation by the effector cells immune system promotes tumor progression and metastasis [2, 3]. Commonly, the mechanisms of tumor escape include, evading the recognition by the immune cells of the host, decreased leukocyte functions (immune suppressive mediators), apoptosis of immune cells, exploitation of immune checkpoints, myeloid derived suppressor cells expansion, expansion of Treg cells, apoptosis evasion by tumor cells, and M2 macrophages expansion. Due to neoplastogenesis, another important factor of tumor development is prevention of the immune response enabled by Treg cells. 
These cells, as a heterogeneous fraction of lymphocytes, function to adjust the immune system. In humans, they comprise $5-10 \%$ of the population of peripheral CD4+ T lymphocytes and $1-2 \%$ of whole lymphocytes ${ }^{4,5}$. One of the most significant subpopulations of them is CD4+CD25+ T lymphocytes also expressing the intracellular Forkhead box P3 (Foxp3) protein [6, 7]. A significant feature of Treg cells is the stable expression of integral membrane protein of $\alpha$ subunit of the IL-2 receptor (IL-2R $\alpha$ ) called CD25. In contrast, CD4+ and CD8+ T lymphocytes display transient expression of this cell surface marker [8]. They are component of the mechanism preventing selectively active cells with autoreactive features and inhibit the expansion of the processes of autoimmune reaction. However, these $T$ lymphocytes may exploit a negative effect, preventing the anti-tumor immune response $[9,10]$. Increased numbers of Treg cells exist inside the tumor, in the vicinity of the tumor, in tumoral draining lymph nodes and in the patients' peripheral blood suffering from various kinds of cancers. There are a number of mechanisms pertained to the Treg cell reposition in tumor masses. Treg cells may be engaged by tumors via hypoxia-provoked chemokines generated by tumor cells, for example C-C motif chemokine ligand 28 (CCL28) and C-C motif chemokine ligand 22 (CL22). Severe propagation of Treg cells inside the tumor mass is the second mechanism. Indeed, the presence of Ki- 67 protein in tumor infiltrating Treg cells may promote proliferation of Treg cells inside the tumor mass. Furthermore, a subset of Treg cells can be proliferated de novo in the presence of TGF $\beta$ and IL-10 within the tumor microenvironment [11, 12]. FoxP3 provides for researchers a useful marker to assess Treg purity, but being an intracellular marker, it is not appropriate for sorting of viable cells $s^{4}$. A number of methods to identify and sort Tregs within a $\mathrm{CD}_{4}^{+} \mathrm{T}$ lymphocyte population are based upon high expression levels of CD25. These procedures can lead to isolation of Treg population with high purity, but the recovery of Tregs is low and time-consuming. Tregs also possess a low level of CD127 and this characteristic can lead to a pure $\mathrm{CD} 4{ }^{+} \mathrm{CD} 25^{+}$Treg population with higher recoveries but currently is not a cost efficient method [13]. Due to the problems mentioned above, including, but not limited to; low population of Tregs in peripheral blood, low recovery methods for Treg isolation, time-consuming and cost ineffective methods, the introduction of a genetically engineered cell line, HEK293, expressing cellular surface marker of $\mathrm{CD} 25$ can be useful. It can be used for the developing assay with the antagonistic therapeutic agents like siRNAs, small molecules, peptides and chimeric antigen receptor (CAR) against CD25 in the conditions of in vitro cell culture for the studies focused on the binding of IL-2 to CD25. Using this approach, the bioinformatics analysis of CD25 RNA secondary structure to explore its stability and in vitro CD25 expression on the cellular surface of HEK293 were accomplished for the first time. Thus, this available cellular model of $\mathrm{CD} 25$ positive can be applicable to facilitate the in vitro developing assays.

\section{Materials and Methods}

Informed consent: Blood samples were collected from healthy volunteers. Informed consent has been obtained from all individuals included in this study.

Ethical approval: The research related to human use has been complied with all the relevant national regulations, institutional policies and in accordance the tenets of the Helsinki Declaration, and has been approved by research ethics committee of University of Isfahan [14].

\section{Computational approach of the RNA structure}

In silico analysis of RNA structure was performed on the RefSeq CDS of CD25 (NM_000417) by RNAfold web server (http://rna.tbi.univie.ac.at/cgi-bin/RNAWebSuite/ RNAfold.cgi) [15, 16].

\section{Isolation and culture of peripheral blood mononuclear cells (PBMCs)}

PBMCs were obtained from healthy blood donors by centrifugation on the Ficoll-Paque (Pharmacia Co., New Jersey, USA) gradient. In brief, $4 \mathrm{ml}$ of Ficoll-Paque gradient was added into two $15 \mathrm{ml}$ centrifuge tubes. The heparinized blood was diluted 1:1 in phosphatebuffered saline (PBS) (Biowest, France) and carefully covered above the Ficoll-Paque gradient ( 9 to $10 \mathrm{ml} /$ tube). The tubes were centrifuged by $1,020 \mathrm{~g}$ for $20 \mathrm{~min}$. The buffy coat layer was gathered carefully, and the cells were washed twice in PBS (by 640g centrifugation for 10 min followed by $470 \mathrm{~g}$ centrifugation for $10 \mathrm{~min}$ ). Then, $1 \times 10^{6}$ cells/ml PBMCs transferred into each T-25 flask. The complete RPMI 1640 cell culture medium (Biowest, France) completed by 10\% fetal bovine serum (FBS) (Biowest, France) and 1\% penicillin-streptomycin (Pen Strep) solution (Gibco, Germany) was used. The mitogenstimulated blasts were obtained by $72 \mathrm{~h}$ culture with $2 \mu \mathrm{g} /$ $\mathrm{ml}$ of phytohemagglutinin-M (PHA-M) (Roche) and the flasks kept at $37^{\circ} \mathrm{C}$ in a humidified incubator with $5 \% \mathrm{CO}$. 


\section{Extraction of RNA from PBMCs}

The whole RNA of stimulated PBMCs was extracted utilizing TRIzol reagent (Invitrogen, Carlsbad, CA, USA) according to the instruction of manufacturer and digested with recombinant DNase I (RNase-free, Takara, Otsu, Japan) to eliminate the probable genomic DNA impurity. Then, the total RNA was aliquoted, stored at $-80^{\circ} \mathrm{C}$ or applied for cDNA synthesis. Prior to the cDNA synthesis, the concentration of whole RNA was identified via NanoDrop 2000C (Thermo Scientific, USA).

\section{RT-PCR}

The RevertAid First Strand cDNA Synthesis Kit (Thermo Fisher Scientific Inc., MA, USA) was utilized to synthesize first strand cDNA by oligo (dT) primer, based on the instruction of manufacturer. The reaction of cDNA synthesis was carried out by the Primus 25 advanced thermal cycler (Peqlab Biotechnologie Gmbh, Erlangen, Germany). The result of reverse transcription was utilized as the template for PCR reaction or was stored at $-20^{\circ} \mathrm{C}$.

\section{PCR}

The first-round PCR reaction contained cDNAs as the DNA template, $25 \mu \mathrm{l}$ Taq DNA Polymerase 2X Master Mix Red with $2 \mathrm{mM} \mathrm{MgCl} 2$ (Amplicon, Denmark), $10 \mathrm{pmol} / \mu \mathrm{l}$ of the first forward primer (F1: ATGGATTCATACCTGCTGATGTGG), and $10 \mathrm{pmol} / \mu \mathrm{l}$ of the first reverse primer (R1: CTAGATTGTTCTTCTACTCTTCCTCTG) in a final volume of $50 \mu \mathrm{l}$. Start codon and stop codon were shown as the bold sequences. Amplification was performed on a Eppendorf Mastercycler Gradient (Eppendorf AG, Hamburg, Germany) with the following program: $95^{\circ} \mathrm{C}$ by $5^{\prime}$ for initial denaturation, $95^{\circ} \mathrm{C}$ by $25^{\prime \prime}, 60^{\circ} \mathrm{C}$ by $30^{\prime \prime}, 72^{\circ} \mathrm{C}$ by $30^{\prime \prime}$ for 30 cycles and final extension of $72^{\circ} \mathrm{C}$ by $5^{\prime}$. Afterwards, the first-round PCR products were diluted 1:100 to add to the second-round PCR reaction as the DNA template. The second forward primer with HindIII restriction site (F2: CCCAAGCTTGGGGCCACCATGGATTCATACCT) and the second reverse primer with EcoRI restriction site (R2: CCGGAATTCCGGCTAGATTGTTCTTCTACTCTTC) was utilized. A Kozak sequence was placed 3' of the HindIII enzyme restriction site in the second forward primer (bold sequence). Underlined sequences showed HindIII and $E c o R I$ restriction site in the second forward and reverse primers, respectively. The surrounding sequences around the restriction sites are named the cleavage close to the end of DNA fragments (oligonucleotides) (recommended by New England Biolabs Co.) for the enzymatic digestion efficiency by the restriction endonucleases. A stop codon was placed 3' of the EcoRI restriction site in the second reverse primer, shown by bold sequence. The other conditions of the second-round PCR including reaction concentration and PCR program are the same. Final PCR yields were electrophoresed by $1 \%(\mathrm{w} / \mathrm{v})$ agarose gel in TBE buffer (0.09 mM Tris, $0.09 \mathrm{mM}$ Boric acid and $20 \mathrm{mM}$ EDTA, pH 8.3), apparent with Ethidium Bromide $(0.5 \mu \mathrm{g} /$ $\mathrm{ml}$ ) and imaged.

\section{Construction of $\mathrm{CD} 25$ expression plasmid}

Plasmid pcDNA3.1(+) (Invitrogen, Grand Island, NY) and CD25 amplicons were singularly digested with HindIII and EcoRI enzymes (Thermo Fisher Scientific Inc., MA, USA). The digested products of the backbone of pcDNA3.1(+) was separated on $1 \%(\mathrm{w} / \mathrm{v})$ agarose gel and purified from the gel utilizing PrimePrep Gel Purification Kit (GeNet Bio Co., Daejeon, South Korea). The digested and linearized pcDNA3.1(+) and digested CD25 amplicons were ligated by T4 DNA ligase enzyme (Thermo Fisher Scientific, Inc., MA, USA) based on the instruction of manufacturer. Ligation outcome was transformed into the chemically competent $E$. coli TOP 10 utilizing heat shock procedure. The pcDNA3.1(+) includes ampicillin resistance gene, thus, E. coli TOP 10 bacteria could grow in LB medium with ampicillin as a selectable antibiotic. Finally, plasmid extraction was done by PrimePrep Plasmid DNA extraction kit (GeNet Bio Co., Daejeon, South Korea).

\section{RFLP analysis, colony PCR and sequencing}

The initial confirmation of the presence of the insert in the obtained clones was determined by a HindIII and EcoRI double digestion in an ultimate volume of $10 \mu \mathrm{l}$ and with the following reaction: $0.5 \mu \mathrm{l}$ of HindIII (5U), $0.25 \mu \mathrm{l}$ of EcoRI (2.5U), $2 \mu \mathrm{l}$ of Tango Buffer (2X), $6.25 \mu \mathrm{l}$ of DDW and $1 \mu \mathrm{l}$ plasmid $(200 \mathrm{ng} / \mu \mathrm{l})$ in $2 \mathrm{~h}$ and $37^{\circ} \mathrm{C}$. The enzymatic inactivation was according to the manufacturer's instruction. To amplify the insert from the recombinant plasmids, the colony PCR was carried using the first forward primer (F: ATGGATTCATACCTGCTGATGTGG) and the first reverse primer (R: CTAGATTGTTCTTCTACTCTTCCTCTG). To verify certainly the transformed clones, DNA sequencing was done by Macrogen Co. (South Korea) using above mentioned the first forward primer and the first reverse primer. 


\section{Cell culture and transfection}

Dulbecco's Modified Eagle's Medium (DMEM) (Atocel, Budapest, Hungary) was used for HEK293 cell culture. It was supplemented with 10\% FBS and 1\% Pen Strep solution. Then, the cultured cell line was kept in a humidified incubator at $37^{\circ} \mathrm{C}(5 \% \mathrm{CO})$. The cells were seeded in a 12-multiwell plate and transfected with $60-70 \%$ confluency. For the transfection of six wells of cells, $1.6 \mu \mathrm{g}$ for each recombinant pcDNA3.1(+) and mock vector, and $4 \mu \mathrm{l}$ Lipofectamine 2000 Reagent (Thermo Fisher Scientific Inc., MA, USA) were provided in $1 \mathrm{ml}$ DMEM and incubated for 20 min based on the instruction of manufacturer. Then, the mixture was utilized to the cell culture wells and incubated for 4-6 h. Afterwards, the media were changed and the cells were used within $48 \mathrm{~h}$ for RNA extraction. The remaining cells were treated with $300 \mathrm{mg} / \mathrm{L}$ Neomycin (Sigma Aldrich, Germany) for 17 days. Finally, CD25 expression at the protein level was evaluated on the cellular surface.

\section{qRT-PCR}

Expression of CD25 was surveyed by Real-time PCR. The cells were harvested and RNA extraction and previously mentioned cDNA synthesis was done. Specific primers for CD25 (NM_000417) (F: GGAGACAGAGGAAGAGTAGAAG and R: AGGCAAGCACAACGGATG) and EEF1A1 (NM_001402) (F: CCCTTCTGGCTTACACACT and R: TGAACCAAGGCATGTTAGCAC) were designed using AlleleID 7.7 software. Quantitative Real-Time PCR was done in triplicate for each sample as well as a non template control (NTC) using Real-Time PCR Master Mix (2X) with high Rox reference dye (Biofact Co., South Korea) on Step-One Real-Time thermal cycler (ABI, USA). The PCR mixture was including $10 \mu \mathrm{l}$ of 2x SYBR Green Master Mix, $10 \mathrm{pmol} / \mu \mathrm{l}$ of each primer and $1 \mu \mathrm{l}$ of cDNA (50 ng/ $\mu \mathrm{l}$ ) in ultimate volume of $20 \mu \mathrm{l}$. PCR amplification was performed by initial denaturation of $95^{\circ} \mathrm{C}$ by $15^{\prime}$, followed by 40 cycles of $95^{\circ} \mathrm{C}$ by $20^{\prime \prime}, 58^{\circ} \mathrm{C}$ by $35^{\prime \prime}$ and $72^{\circ} \mathrm{C}$ by $30^{\prime \prime}$ and final extension of $72^{\circ} \mathrm{C}$ by $3^{\prime}$. The specificity of the amplifications was identified, according to the melting curves produced by heating the amplicons from 55 to $95^{\circ} \mathrm{C}$. CD25 expression level was evaluated by EEF1A1 housekeeping gene. According to the $2^{-\Delta \Delta C t}$ method, the relative expression of $\mathrm{CD} 25$ gene was measured.

\section{Flow cytometry}

Fluorescence-activated cell sorting (FACS) using CD25 monoclonal antibody (BC96), conjugated with phycoerythrin (PE) and mouse IgG1 kappa Isotype control, conjugated with PE (eBioscience ${ }^{\mathrm{TM}}$, USA) were applied. The CD25 expressing HEK293 cells, the mock transfected HEK293 as well as untransfected HEK293 cells (as negative control) were utilized for flow cytometric technique. Nearly, $5 \times 10^{5}$ cells were washed three times and re-suspended in a total volume of $100 \mu \mathrm{l}$ PBS. $1 \mu \mathrm{g}$ of each monoclonal antibody was mixed, and cells were kept for $30 \mathrm{~min}$ in the room temperature (RT) and darkness. After washing with PBS, cells were evaluated with Cell Quest Pro software in a FACS Callibour (BD Biosciences, USA) apparatus.

\section{Statistical analysis}

Statistical analysis was performed by IBM SPSS Statistics V21.0 (SPSS Inc., Chicago, IL, USA) through parametrical test. Paired-T-Test and one way ANOVA were utilized to compare the outcomes before and after interventions between the groups. As the statistical significance, $\mathrm{p}<$ 0.05 was considered.

\section{Results}

\section{Secondary RNA structure analysis}

The calculated minimum free energy (MFE), the free energy of the thermodynamic ensemble and the centroid secondary structure with a MFE of CD25 RNA were $-246.70 \mathrm{kcal} / \mathrm{mol},-258.66 \mathrm{kcal} / \mathrm{mol}$ and $-187.90 \mathrm{kcal} / \mathrm{mol}$, respectively. In addition, the MFE secondary structure, the base-pairing probability and the mountain plot for CD25 were depicted (Figure 1a, Figure 1b and Figure 1c).

\section{Cloning of CD25 into pCDNA3.1(+)}

RNA isolation from PHA-stimulated blasts of PBMCs (Figure 2a) was successfully performed. cDNA of CD25 was amplified successfully by the first- and the secondround PCR to add overhangs (Figure 2b, Figure 2c). PCR products showed a 837 bp nucleic acid fragment of CD25 cDNA. In order to remove unspecific bands from cDNA products, the extra gel purification step was done (Figure 


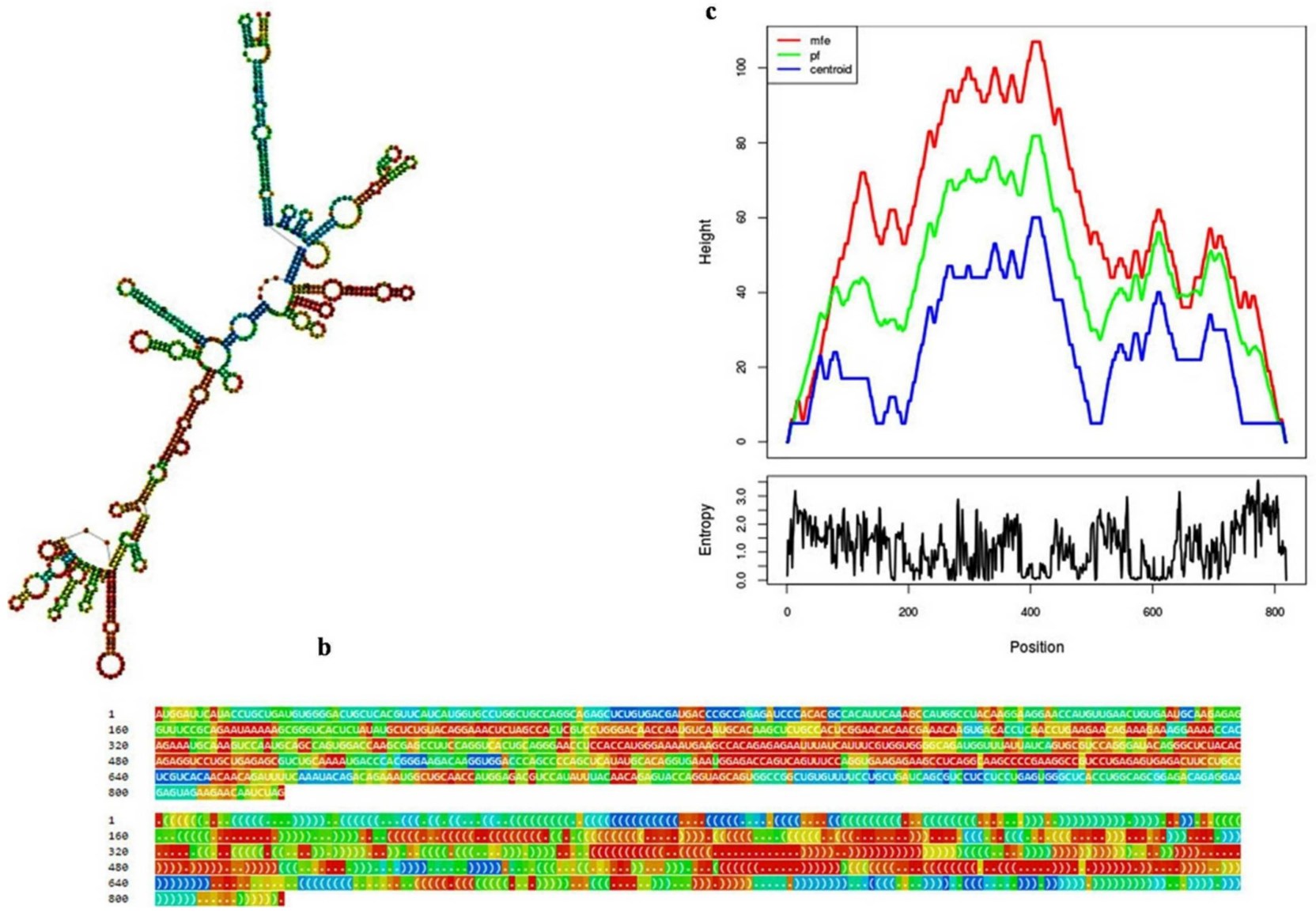

Figure 1: (a) The MFE secondary structure, (b) the base-pairing probability and (c) the mountain plot for CD25 (the MFE structure (red), the thermodynamic ensemble of RNA structures (green) and the centroid structure (blue)) were obtained. Under the mountain plot, positional entropy was given.

3a). The pcDNA3.1(+) plasmid and specific cDNA fragment of $\mathrm{CD} 25$ were digested by HindIII and EcoRI restriction endonucleases (Figure $3 \mathrm{~b}$ ) and then, they were ligated using T4 DNA ligase. After bacterial transformation, verification of the successful procedure was performed by colony PCR (Figure 3c), and RFLP analysis (Figure 3d) and finally, by sequencing (Figure 3e). Using NCBI blast, the sequencing result of the coding sequence (CDS) of CD25 with 100\% identity among three human transcript variants was approved (Figure 3f).

\section{Transcript expression level of CD25}

As indicated in Figure 4a, transcript level of CD25 was measured in transfected, mock and untransfected HEK293 cells with qRT-PCR. CD25 mRNA increased nearly five-fold in comparison with negative control and mock $(p<0.05)$ (Figure 4a).

\section{Protein expression level of CD25}

The cells were analyzed using anti CD25-PE antibody for CD25 expression. In comparison with untransfected control, flow cytometry analysis showed that $76.2 \%$ of transfected cells were expressing CD25 protein $(p<0.05)$ (Figure 4b). As it is indicated in Figure 4b, there was no significant difference among negative control and mock for CD25 expression which confirmed that the cellular surface expression was specific.

\section{Discussion}

Nowadays, cellular models play an important role in basic medical laboratory researches. These models can help scientists to find clues pertained to pharmaceutical effects in cell culture conditions, before the animal experiments and clinical trials in human patients. Several 

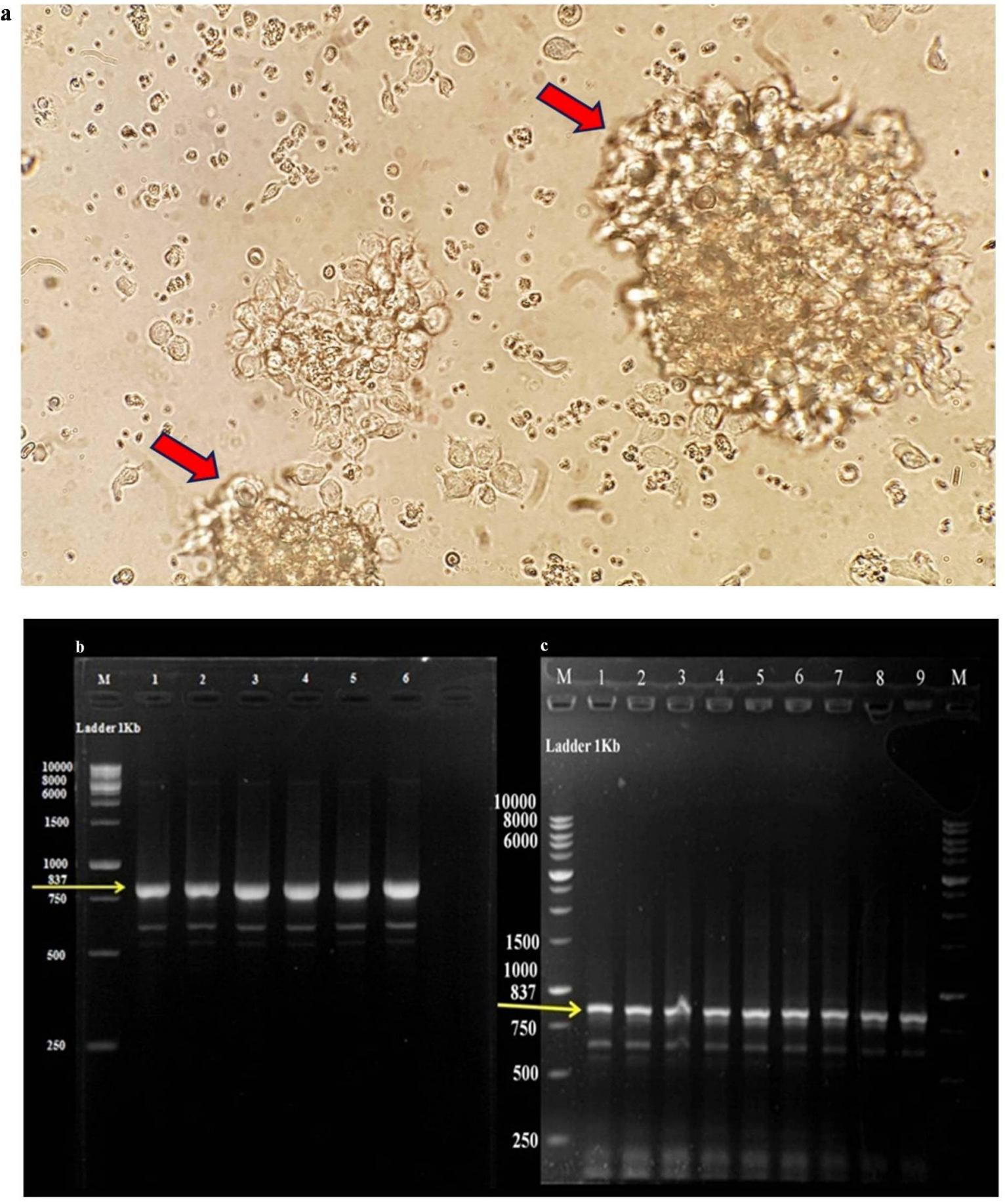

Figure 2: (a) PHA-stimulated blasts of PBMCs were cultured 72h and observed by XDS-1 inverted microscope (25X) (Chongqing COIC Industrial Co., China). The blasts of PBMCs were marked by two red arrows. (b) First round-PCR containing cDNAs as the templates. The wells of 1 to 6 were amplified in the annealing temperatures of $55.6,56.8,58.1,59.5,60,62.1^{\circ} \mathrm{C}$, respectively. There were no great differences among the amplified PCR products due to the different annealing temperatures. (c) Second round-PCR containing first round-PCR products as the templates. The wells of 1 to 9 were amplified in the annealing temperatures of $60,61.6,62.8,64.1,65.5,66.9,68.1,69.2,70.5^{\circ} \mathrm{C}$ respectively. There were no great differences among the amplified PCR products due to the different annealing temperatures. In both agarose gels, the wells were marked by “M” referred to GeneRuler 1Kb DNA Ladder (Cat No. SM0311, Thermo Fisher Scientific Inc., MA, USA). Also, the wells were marked by the numbers referred to the same reactions in the different annealing temperature by gradient PCR. 

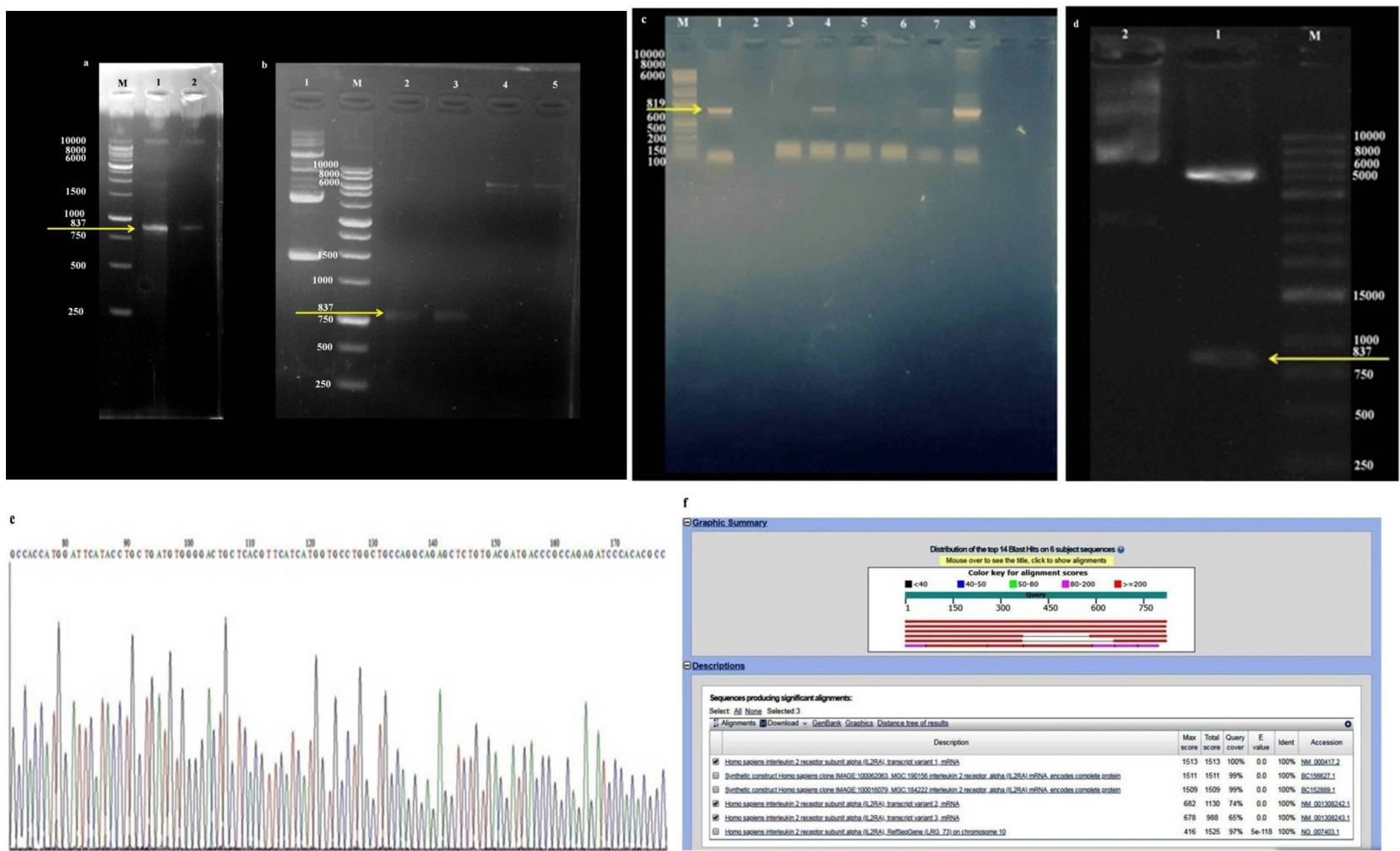

Figure 3: (a) Due to the unspecific bands from previous PCR step, the extra gel purification was performed to excise and purify the band of $C D 25$ coding sequence based on the selected annealing temperature of $60^{\circ} \mathrm{C}$. The well was marked by " $M$ " referred to GeneRuler $1 \mathrm{~Kb}$ DNA Ladder (Thermo Fisher Scientific Inc., MA, USA). Also, the wells were marked by the numbers of 1 and 2 referred to the different concentrations of excised band from two repeats. (b) Digested pcDNA3.1(+) plasmid and specific cDNA fragment of CD25 by HindIII and EcoRI. The well was marked by "M" referred to GeneRuler 1Kb DNA Ladder (Thermo Fisher Scientific Inc., MA, USA). Also, the wells were marked by the numbers of 1, 2, 3, 4 and 5 referred to the undigested plasmid of pcDNA3.1(+), double digested of coding sequence of CD25 fragment (wells of 2 and 3) and double digested of pcDNA3.1(+) plasmid (wells of 4 and 5), respectively. It is noticeable that the bands of $837 \mathrm{bp}$ in both images were made by digestion by considering the accurate digestion sites. (c) Verification of the successful procedure was performed by colony PCR. In this image, "M" was referred to KAPA Universal DNA Ladder Kit (Kapa Biosystems, Inc., Wilmington, USA) and band of CD25 with $819 \mathrm{bp}$ in length was amplified by PCR with the first forward and the first reverse primers. The wells of $1,4,7$ and 8 pertained to the colonies possessed recombinant plasmids. Other wells pertained to the non-recombinant colonies. There were primerdimer accumulations below the lanes. (d) RFLP analysis on the bacterial colony possessed recombinant plasmid. The well was marked by "M" referred to GeneRuler 1Kb DNA Ladder (Thermo Fisher Scientific Inc., MA, USA). The well 1 and 2 referred to the double digested recombinant plasmid and undigested recombinant plasmid, respectively. (e) Apart of the sequencing result of the CD25 insert from the recombinant plasmid. (f) Using NCBI blast, the sequencing result of the coding sequence of CD25 by 819 nucleotides in length was approved with $100 \%$ identity among three human transcript variants. It is noticeable that the resulted fragment of 819 bp in NCBI blast was obtained by considering the accurate coding sequence of CD25.

previous studies showed that PBMCs, due to having same neurotransmitter receptors as in brain tissues, are suitable as cellular models to study the psychiatric disorders [17-19]. Also, they have been used in the studies of immune dysfunction disorders such as type 2 diabetes, obesity $[20,21]$ and for drug screening to analysis the effect of pharmaceutical agents on corresponding signaling pathways [22-24]. PBMCs have been applied for studding the effects of metformin in breast cancer and type 2 diabetes [25], growth hormone treatment [26] and multiple sclerosis [27]. However, PBMCs contain a varied population of immune cells including monocytes, lymphocytes (T Cells, B cells, and NK cells) and dendritic cells. Amongst individuals, the frequencies of ratio of PBMCs subset can be different. Furthermore, as mentioned previously, the study of one important subpopulation of PBMCs, like Treg cells, faces many problems. On the other hand, their importance in cancer investigations requires the cellular models expressing the critical biomarkers. Also, the importance of IL-2 in immune responses and in 
a

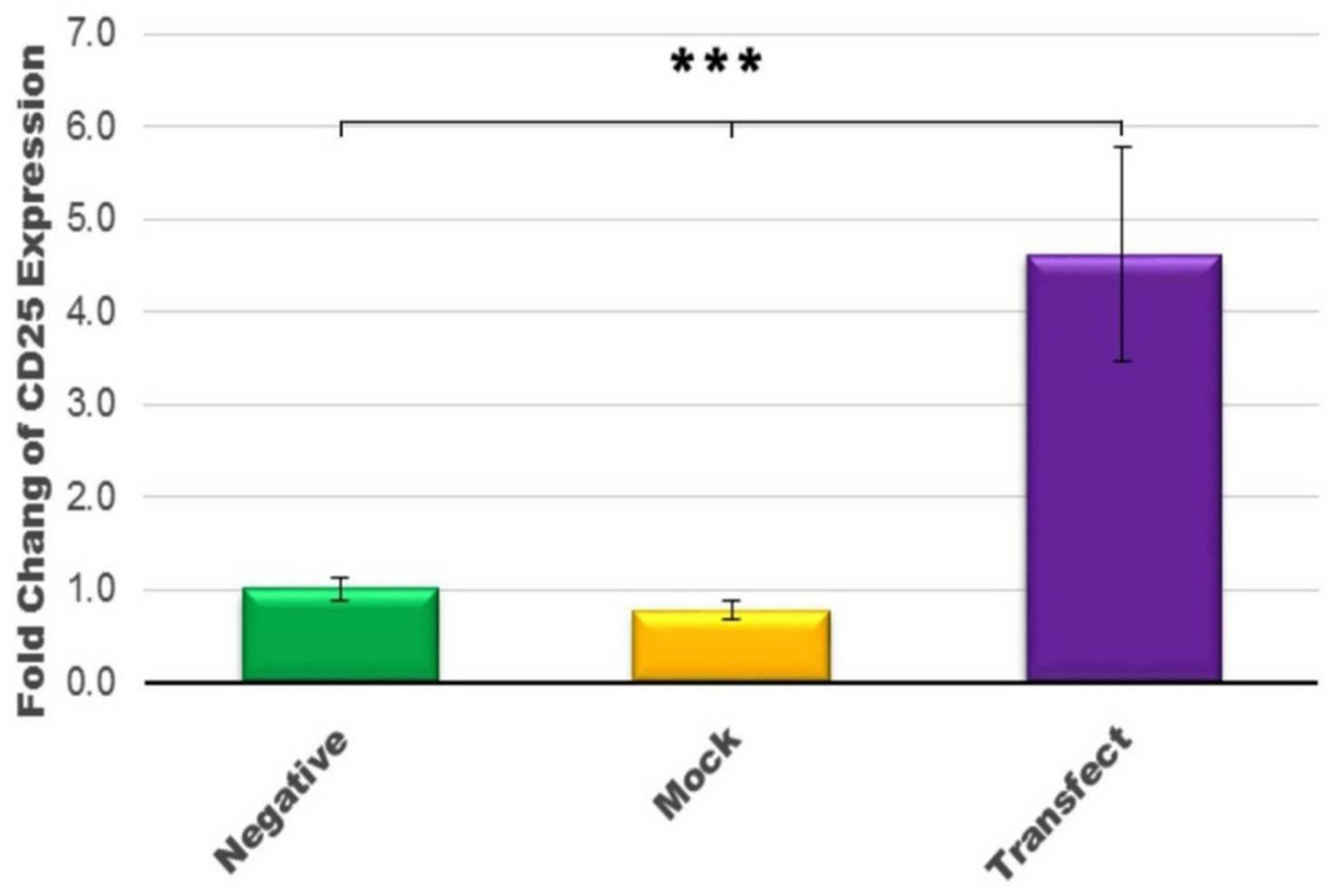

b

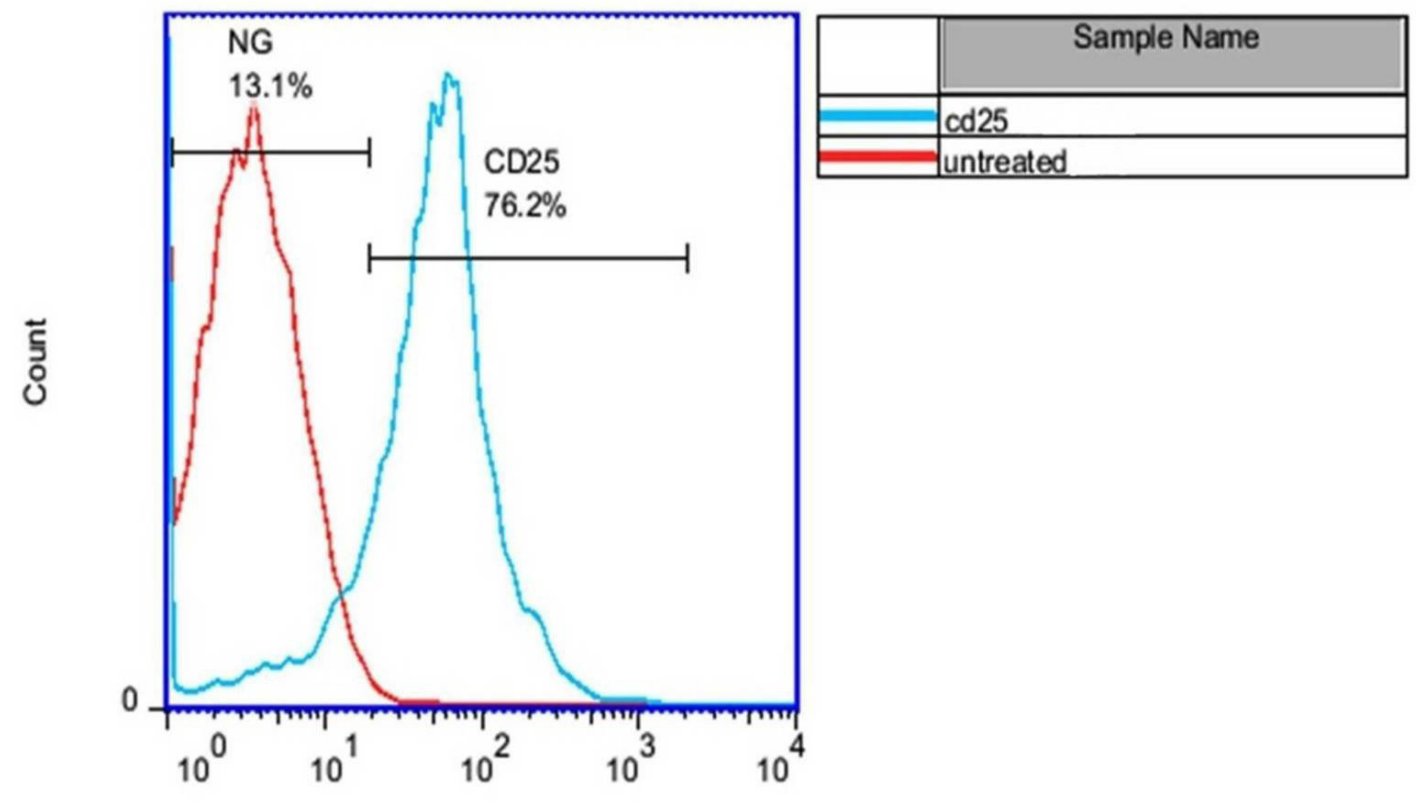

$\mathrm{FL} 2 \cdot \mathrm{H}$

Figure 4: (a) Measurement of CD25 mRNA level in HEK293 cells using qRT-PCR method. CD25 is highly expressed in transfected HEK293 cells while it is almost undetectable in negative control (untransfected) and Mock. Asterisk $\left(^{\star}\right)$ represents statistical significance $(p<0.001)$. Results are shown as mean \pm SEM obtained from three identical repeats of each experiment. (b) Flow cytometry analysis of CD25 expression on the cellular surface of HEK293 cell line. The histogram shows $76.2 \%$ of the cells are expressing CD25. 
several pathogenic conditions like tumors has motivated researches. In spite of existence of commercial monoclonal antibodies, including Daclizumab and Basiliximab that bind to CD25, there is no alternative drug to inhibit directly and efficiently CD25.

From a mechanistic viewpoint, researchers have demonstrated that the formation of the IL-2 receptor complex is triggered by the molecular interaction between IL-2 and CD25, followed by the sequential interaction of CD122 (IL-2Rß) and CD132 (IL-2Rgc) [28]. As the normal renal epithelial derived cell line, HEK293 possess intrinsically functional CD132 and CD122 on their cellular surfaces [2932]. In addition, JAK/STAT signaling pathway, as the main signaling pathway for IL-2, are existed and functioned in HEK293 cell line [33]. Therefore, in this article, the initial and essential step forming the receptor complex of IL-2 meaning the overexpressed CD25 was considered. By hindering the formation of the CD25/IL-2, suppression of the signaling of IL-2R in Treg cells using antagonists might be achievable to treat cancers in combination therapy with other pharmaceutical agents.

In case of secondary structure analysis of RNA, based on the thermodynamic theory, the most negative value of free energy is valued as the most structured and the most stable RNA. Therefore, the secondary structure of CD25 was considered and the MFE of $-246.70 \mathrm{kcal} / \mathrm{mol}$ was resulted. The average base-pair distance between all structures in the thermodynamic ensemble known as the ensemble diversity. In this regard, the RNA of CD25 had the score of 220.63 as the ensemble diversity. Also, the free energy of the thermodynamic ensemble was -258.66 $\mathrm{kcal} / \mathrm{mol}$. The structure with the minimum total basepair distance than all structures in the thermodynamic ensemble called as the centroid structure. The centroid secondary structure with a minimum free energy of -187.90 $\mathrm{kcal} / \mathrm{mol}$ was gained. The mountain plot (including the MFE structure, the thermodynamic ensemble of the RNA structure and the centroid structure) demonstrates the secondary structure of CD25 mRNA in a plot of height against position, where the height is represented by the number of base pairs encompassing the base at a specific position ${ }^{15}$. In fact, in this representation, loops pertain to plateaus and hairpin loops include peaks. Additionally, the positional entropy for each position is represented below the mountain plot [16]. Regarding the CD25 mRNA results, the high negative value of above mentioned MFE demonstrated that this structure could be the stable RNA structure. The expression of CD25 was explored using qRTPCTR finding the transfected cell line to have higher levels of CD25 than the mock-transfection and the negative control. This observation was confirmed at the protein level using FACS, finding similar results. In this way, this important marker was recognized by flow cytometry technique with $76.2 \%$ protein expression level on the cellular surface of HEK293.

The purpose of producing HEK293 cells expressing CD25 on the cellular surface was to introduce an available cellular tool for developing assays of synthetic or biopharmaceutical drugs to enhance studies of human Treg cells in in vitro cell culture for the studies focused on the binding of IL-2 to CD25. Accordingly, the purpose was fulfilled and the efficient cellular model was introduced.

Acknowledgments: The authors are thankful for The Graduate Office of University of Isfahan to financially support the project (Grant No. 8489). Also, the authors wish to thanks to Vida Homayouni, Mohadeseh Toghyani and Fahimeh Hoseininasab for their technical helps. '

\section{Conflict of interest: Authors state no conflict of interest}

Declaration: The authors alone are responsible for the content of this manuscript.

\section{References}

1. Yang Y. Cancer immunotherapy: harnessing the immune system to battle cancer. Journal of Clinical Investigation. 2015;125(9):3335-3337.

2. Gastman BR, Johnson DE, Whiteside TL, Rabinowich H. Tumorinduced apoptosis of T lymphocytes: elucidation of intracellular apoptotic events. Blood. 2000;95(6):2015-2023.

3. Kim R, Emi M, Tanabe K. Cancer immunosuppression and autoimmune disease: beyond immunosuppressive networks for tumour immunity. Immunology. 2006;119(2):254-264.

4. Baecher-Allan C, Brown JA, Freeman GJ, Hafler DA. CD4+CD25high regulatory cells in human peripheral blood. Journal of Immunology. 2001;167(3):1245-1253.

5. Ahmadzadeh M, Felipe-Silva A, Heemskerk B, Powell DJ Jr, Wunderlich JR, Merino MJ, et al. FOXP3 expression accurately defines the population of intratumoral regulatory $T$ cells that selectively accumulate in metastatic melanoma lesions. Blood. 2008;112(13):4953-4960.

6. Garden OA, Pinheiro D, Cunningham F. All creatures great and small: regulatory $T$ cells in mice, humans, dogs and other domestic animal species. International Immunopharmacology. 2011;11(5):576-588.

7. Jagła M, Cichocka-Jarosz E. Limfocyty regulatorowe. Alergia Astma Immunologia. 2007;12:22-29.

8. Ryba M, Myśliwska J. CD4+CD25+Foxp3+ T lymphocytes: naturally occuring regulatory T cells. Pediatric Endocrinology Diabetes and Metabolism. 2010;16(4):289-294.

9. Biller BJ, Elmslie RE, Burnett RC, Avery AC, Dow SW. Use of FoxP3 expression to identify regulatory $T$ cells in healthy 
dogs and dogs with cancer. Veterinary Immunology and Immunopathology. 2007;116(1-2):69-78.

10. Qin FX. Dynamic behavior and function of Foxp3+ regulatory T cells in tumor bearing host. Cellular \& Molecular Immunology. 2009;6(1):3-13.

11. Beyer M, Schultze JL. Regulatory T cells: major players in the tumor microenvironment. Current Pharmaceutical Design. 2009;15(16):1879-1892.

12. Oleinika K, Nibbs RJ, Graham GJ, Fraser AR. Suppression, subversion and escape: the role of regulatory $T$ cells in cancer progression. Clinical and Experimental Immunology. 2013;171(1):36-45.

13. Boyce C, Lane Y, Hingorani R, McIntyre C, Ruitenberg J, Ghanekar S. Human Regulatory T-Cell Isolation and Measurement of Function. BD Biosciences, Application Notes. (2010).

14. van der Kooi-Pol MM, Sadaghian Sadabad M, Duipmans JC, Sabat AJ, Stobernack T, Omansen TF, et al. Topography of distinct Staphylococcus aureus types in chronic wounds of patients with epidermolysis bullosa. PLoS One. 2013;8(6):e67272.

15. Lorenz R, Bernhart SH, Höner Zu Siederdissen C, Tafer H, Flamm C, et al. ViennaRNA Package 2.0. Algorithms for Molecular Biology. 2011;6:26.

16. Gruber AR, Lorenz R, Bernhart SH, Neuböck R, Hofacker IL. The vienna RNA websuite. Nucleic Acids Research. 2008;36:W70W74.

17. Gladkevich A, Kauffman HF, Korf J. Lymphocytes as a neural probe: potential for studying psychiatric disorders. Progress in Neuro-Psychopharmacology \& Biological Psychiatry. 2004;28(3):559-576.

18. Torres KC, Souza BR, Miranda DM, Nicolato R, Neves FS, Barros $A G$, et al. The leukocytes expressing DARPP32 are reduced in patients with schizophrenia and bipolar disorder. Progress in Neuro-Psychopharmacology \& Biological Psychiatry. 2009;33(2):214-219.

19. Rollins B, Martin MV, Morgan L, Vawter MP. Analysis of whole genome biomarker expression in blood and brain. American Journal of Medical Genetics. 2010;153B(4):919-936.

20. Cortez-Espinosa N, Cortés-Garcia JD, Martínez-Leija E, Rodríguez-Rivera JG, Barajas-López C, González-Amaro R, et al. D39 expression on Treg and Th17 cells is associated with metabolic factors in patients with type 2 diabetes. Human Immunology. 2015;76(9):622-630.

21. Mahmoud F, Al-Ozairi E, Haines D, Novotny L, Dashti A, Ibrahim $B$, et al. Effect of Diabetea tea ${ }^{\mathrm{TM}}$ consumption on inflammatory cytokines and metabolic biomarkers in type 2 diabetes patients. Journal of Ethnopharmacology. 2016;194:1069-1077.

22. Jawa V, Cousens LP, Awwad M, Wakshull E, Kropshofer H, De Groot AS. T-cell dependent immunogenicity of protein therapeutics: preclinical assessment and mitigation. Clinical Immunology. 2013;149(3):534-555.

23. Becker K, Schroecksnadel S, Gostner J, Zaknun C, Schennach H, Uberall $F$, et al. Comparison of in vitro tests for antioxidant and immunomodulatory capacities of compounds. Phytomedicine. 2014;21(2):164-171.

24. Wesseling H, Guest PC, Lago SG, Bahn S. Technological advances for deciphering the complexity of psychiatric disorders: merging proteomics with cell biology. International Journal of Neuropsychopharmacology 2014;17(8):1327-1341.
25. Damjanović A, Matić IZ, Đorđić M, Đurović MN, Nikolić S, Roki K, et al. Metformin effects on malignant cells and healthy PBMC; the influence of metformin on the phenotype of breast cancer cells. Pathology \& Oncology Research. 2015;21(3):605-612.

26. Welzel M, Appari M, Bramswig N, Riepe FG, Holterhus PM. Transcriptional response of peripheral blood mononuclear cells to recombinant human growth hormone in a routine fourdays IGF-I generation test. Growth Hormone \& IGF Research. 2011;21(6):336-342.

27. Valenzuela RM, Kaufman M, Balashov KE, Ito K, Buyske S, DhibJalbut S. Predictive cytokine biomarkers of clinical response to glatiramer acetate therapy in multiple sclerosis. Journal of Neuroimmunology. 2016;300:59-65.

28. Rickert M, Wang X, Boulanger MJ, Goriatcheva N, Garcia KC. The structure of interleukin-2 complexed with its alpha receptor. Science. 2005;308(5727):1477-1480.

29. Benitah SA, Valeron PF, Rui H, Lacal JC. STAT5a activation mediates the epithelial to mesenchymal transition induced by oncogenic RhoA. Molecular Biology of the Cell. 2003;14(1):40 53.

30. Tejman-Yarden N, Zlotnik M, Lewis E, Etzion O, Chaimovitz C, Douvdevani A. Renal cells express a functional interleukin-15 receptor. Nephrology Dialysis Transplantation. 2005;20(3):516523.

31. Tsukadaira A, Okubo Y, Koyama S, Sato E, Nagase H, Agematsu $\mathrm{K}$, et al. Human bronchial epithelium expresses interleukin-9 receptors and releases neutrophil chemotactic factor. Experimental Lung Research. 2002;28(2):123-139.

32. Boyman 0, Sprent J. The role of interleukin-2 during homeostasis and activation of the immune system. Nature Reviews Immunology. 2012;12(3):180.

33. Kuniyasu H, Ohmori H, Sasaki T, Sasahira T, Yoshida K, Kitadai Y, et al. Production of interleukin 15 by human colon cancer cells is associated with induction of mucosal hyperplasia, angiogenesis, and metastasis. Clinical Cancer Research. 2003;9(13):4802-4810. 